\title{
CRESCIMENTO DE PLANTAS DE CAJ UEIRO ANÃO PRECOCE INFLUENCIADO PELA COMPACTAÇÃO E TEMPERATURA DO SOLO ${ }^{(1)}$
}

\author{
P. K. B. OLIVEIRA(2), T. S. OLIVEIRA(3), \\ A. M. CAMELO ${ }^{(4)} \&$ R. E. ROMERO ${ }^{(5)}$
}

\begin{abstract}
RESUMO
Atribui-se ao uso de práticas culturais inadequadas uma das causas da redução drástica dos níveis de produtividade e produção da cultura do caju no nordeste brasileiro. A eliminação periódica da cobertura vegetal e o tráfego de máquinas e implementos condicionam temperaturas e densidade de solo que podem comprometer o crescimento e desenvolvimento das plantas, o que é mais preocupante ainda, considerando a textura arenosa dos solos cultivados e as condições climáticas da região. A partir destas constatações, desenvolveu-se o presente trabalho, com vistas em fornecer indicativos capazes de permitir uma avaliação dos efeitos das condições de solo e clima, objetivando identificar o comportamento de plantas de cajueiro anão precoce, submetidas a diferentes níveis de compactação e temperatura de solo, como meio de testar a hipótese de que níveis crescentes destes fatores podem reduzir seu crescimento. 0 presente trabalho foi desenvolvido em condições controladas, utilizando colunas de solo em tubos de PVC, nas quais se adi cionou o Argissolo Vermelho-Amarelo eutrófico típico. Submeteram-se estas colunas a dois níveis de temperatura do solo, um com as colunas de solo isoladas com folhas de isopor pintadas na cor alumínio (tratamento isolado- $\mathrm{TI}$ ), e o outro, com colunas não isoladas, mas com os tubos de PVC pintados na cor preta, para maior absorção de luz (tratamento não isolado-TNI). Os níveis de densidade a que foram ajustadas as camadas
\end{abstract}

\footnotetext{
(1) Parte da Tese de Mestrado do primeiro autor, apresentada ao Curso de Solos e Nutrição de Plantas, Universidade Federal do Ceará - UFC. Financiado pela FUNCAP. Recebido para publicação em junho de 2001 e aprovado em abril de 2002.

(2) Mestrando em Agronomia, Departamento de Ciências do Solo, Universidade Federal do Ceará - UFC. Bloco 807, Campus do $\mathrm{PICl}, \mathrm{CEP}$ 60455-760 Fortaleza (CE).

(3) Professor Adjunto do Departamento de Ciências do Solo, UFC. Bolsista do CNPq. E-mail: teo@ufc.br

(4) Estudante de Graduação do Curso de Agronomia, UFC.

(5) Professor Assistente do Departamento de Ciências do Solo, UFC. E-mail: reromero@ufc.br
} 
compactadas da coluna de solo foram 1,2; 1,3; 1,4; 1,5; 1,6 e 1,7 $\mathrm{Mg} \mathrm{m}^{-3}$, sendo o restante da coluna a $1,3 \mathrm{Mg} \mathrm{m}^{-3}$. Os tratamentos foram distribuídos em delineamento inteiramente casualizado, com arranjo fatorial $6 \times 2$ (seis níveis de compactação e dois níveis de temperatura), com três repetições. Após o período de 12 meses de cultivo das plantas, a parte aérea foi colhida, determinando-se matéria seca da parte aérea, altura da planta, diâmetro do caule e número de ramificações da parte aérea. Os anéis da coluna de solo foram separados e as raízes foram coletadas, determinando-se, por meio do número de pixels, a área radicular, o comprimento de raízes e a densidade radicular. $\mathbf{0}$ aumento da temperatura e da densidade do solo reduziu o cresci mento, afetando tanto a parte aérea quanto o sistema radicular das plantas de cajueiro.

Termos de indexação: manejo do solo, culturas perenes, sistema radicular, solos arenosos, Formação Barreiras.

\title{
SUMMARY: GROWTH OF CASHEW PLANTS AFFECTED BY INCREASED SOIL DENSITY AND TEMPERATURE
}

\begin{abstract}
I nadequatecrop practices areamong themain causes for reduced productivity of cashew crops in the Northeast of Brazil. Periodical elimination of vegetation and the use of heavy machines and implements infl uencesoil temperatures and density, possibly affecting plant growthand devel opment. This is an aggravating fact, consi dering thesandy soils and cl imate conditions of theregi on. This study had theobjecti veto investigatethedevel opment of cashew nut plants (early-dwarf cultivar) at different levels of soil temperature and density, to corroboratethehypothesis: thehigher thelevel of thesefactors, thelower theexpected plant growth and biomass production. PVC tubes were filled with samples of eutrophic RedYellow Typic Podzolic soil under controlled conditions and these soil col umns exposed to two temperatures. In one of the treatments, PVC columns were insulated with polystyrene sheets and painted in aluminium col or (I nsulati on treatment-IT), and in theother treatment, PVC col umns without sheets were pai nted bl ack to absorb moresunl ight (treatment without insulation-TWI ). Density levels used in the compressed soil layers were 1.2, 1.3, 1.4, 1.5, 1.6, and $1.7 \mathrm{Mg} \mathrm{m}^{-3}$, and $1.3 \mathrm{Mg} \mathrm{m}^{-3}$ in therest of thecolumn. Theexperimental desi gn was completely randomized in three replications, for six density and two temperature level treatments. After twelve months, plant shoots were collected to determine bi omass, height, stem diameter and number of branches. Soil rings and roots were separated to determine the root area, root length, and root density by pixel number. Results indicated that higher soil temperatures and densities reduced growth, affecting both above-ground biomass and roots of the cashew nut plants.
\end{abstract}

Index terms: Soil management, perennial cultures, root systems, sandy soils, Barreiras Formation.

\section{NTRODUÇÃO}

A cultura do caju (Anarcadium occidentale L.) apresenta grande importância para a economia brasileira. O Brasil é um dos principais produtores da amêndoa de castanha de caju ( 25 \% da produção mundial, 1993/1994), cuja produçãoé predominantemente destinada ao mercado externo (97\%). Os principais países importadores da amêndoa de castanha de caju brasileira são os Estados Unidos (85\%), Canadá (6\%), Holanda (3\%) e Alemanha Ocidental (1\%), os quais constituem importante mercado para esse produto (Paula Pessoa et al., 1995).
Nos últimos anos, tem-se constatado, no entanto, uma redução drástica dos níveis de produtividadee produção dessa cultura nas áreas cultivadas (Pimentel et al., 1993). De acordo com esses autores, no período de 1978 a 1990, houve uma redução de 69,66 e $40 \%$ no rendimento médio por hectare, nos estados do Ceará, Piauí e Rio Grande do Norte, respectivamente. Araújo (1991) atribui esta situação ao baixo potencial genético da cultura, à alta incidência de pragas e doenças, à baixa fertilidade dos solos, ao uso de práticas culturais inadequadas e ao uso insuficiente de fertilizantes, corretivos e defensivos. Pimentel et al . (1993) fizeram referências 
à reduzi da oferta de tecnol ogia até meados da década de 80 e à falta de condições financeiras dos produtores para aplicação de tecnologia.

O desenvolvimento vegetal e a produção das culturas agrícolas estão diretamente relacionados com as condições de meio para o estabel ecimento do sistema radicular, além dos fatores ambientais que influenciam o crescimento da parte aérea. Especificamente no caso de culturas perenes, como a cultura do cajueiro, esses fatores são ainda mais expressivos, uma vez que a escala temporal de permanência destas plantas no ambiente, sob a influência direta dos condicionantes climáticos e de solo, é muito maior. O impedimento mecânico, a baixa disponibilidade de oxigênio e nutrientes, a presença de substâncias e elementos tóxicos, a temperatura, a umidade e o ataque de pragas e moléstias (Merten \& Mielniczuk, 1991, citandoArkin \& Taylor, 1981) são alguns dos fatores de solo que devem ser considerados. Dentre os fatores ambientais, a temperatura do solo para crescimento ótimo das plantas varia consideravel mente com o tipo de planta e com as diferentes fases dos processos vegetais (Brady, 1989). As el evadas temperaturas do solo e os períodos que as culturas se submetem a essas temperaturas provocam prejuízos às plantas, contribuindo para o efeito deletério em seu crescimento, desenvol vimento e produção (B ortoluzzi \& Eltz, 2000).

Estudando a exigência da temperatura do solo durante a fase vegetativa e reprodutiva das princi pais culturas agrícolas, M ota (1977) relata que, após a germinação, a temperatura do solo pode ainda influenciar o índice da área foliar das plântulas. Gradual mente, contudo, a temperatura do ar e outros fatores climáticos, como a luz, tornam-se de maior importância para a fase vegetativa e reprodutiva. Para temperaturas quase ótimas, a taxa de absor ção da água pode aumentar na ordem de $10 \%$ para cada grau de aumento na temperatura do solo. Por outro lado, a condução da água nos vasos do xilema às fol has aumenta somente na ordem de $1 \%$ para cada ${ }^{\circ} \mathrm{C}$ de incremento da temperatura do solo. Especificamente para a cultura do cajueiro, não foram encontradas na literatura, referências relativas ao comportamento desta cultura em condições de sol o e clima.

A partir dessas constatações, buscou-se desenvolver o presentetrabalho, objetivando avaliar o comportamento de plantas de cajueiro anão precoce, submetidas a diferentes níveis de compactação e temperatura de sol o.

\section{MATERIAL E MÉTODOS}

O experimento foi realizado no período de abril de 1997 a março de 1998, na área experimental do
Departamento de Fitotecnia da Universidade Federal do Ceará, localizada no Campus do Pici. Foi utilizado um Argissol o Vermelho-Amarelo eutrófico (Quadro 1).

Utilizaram-se colunas de solo formadas pela junção de quatro anéis de PVC, cada um com $20 \mathrm{~cm}$ de comprimento e $15 \mathrm{~cm}$ de diâmetro. Os anéis, justapostos e unidos por fita adesiva, foram preenchidos com material de sol o com densidade de $1,3 \mathrm{Mg} \mathrm{m}^{-3}$, à exceção do segundo anel, cuja densidade do solo foi ajustada aos seis níveis de compactação propostos neste estudo $(1,2 ; 1,3 ; 1,4$; 1,5; 1,6 e 1,7 $\mathrm{Mg} \mathrm{m}^{-3}$ ). Dois níveis de temperaturas de solo também foram testados, simulados com o isolamento das colunas com folhas de isopor e pintura na cor alumínio (tratamento isolado $=\mathrm{TI}$ ) e outro com a pintura das col unas na cor preta, visando à maior absorção de luz (tratamento não isolado = TNI). Os tratamentos foram distribuídos em del ineamento inteiramente casualizado, com arranjo fatorial $6 \times 2$ (seis níveis decompactaçãoe dois níveis de temperatura), com três repetições.

Para a montagem das colunas e obtenção dos níveis de compactação desejados, adotou-se o procedimento proposto por Alvarenga et al. (1996), adaptado para o presente estudo. Para tanto, foram utilizados prensa hidráulica, anéis de PVC de diferentes espessuras e uma camisa de ferro, para

\section{Quadro 1. Atributos físíco e quími $\cos ^{(1)}$ de um Argissolo Vermelho-Amarelo eutrófico típico, coletado no Campus do PICI, Universidade Federal do Ceará}

\begin{tabular}{|c|c|}
\hline Atributo & Resultado \\
\hline $\mathrm{pH}$ & 5,50 \\
\hline $\mathrm{P}\left(\mathrm{mg} \mathrm{kg}^{-1}\right)$ & 8,00 \\
\hline $\mathrm{K}+\left(\mathrm{cmol}_{\mathrm{c}} \mathrm{kg}^{-1}\right)$ & 0,16 \\
\hline $\mathrm{Ca}^{2+}\left(\mathrm{cmol}_{\mathrm{c}} \mathrm{kg}^{-1}\right)$ & 1,30 \\
\hline $\mathrm{Mg}^{2+}\left(\mathrm{cmol}_{\mathrm{c}} \mathrm{Kg}^{-1}\right)$ & 1,00 \\
\hline $\mathrm{Al}^{3+}\left(\mathrm{cmol}_{\mathrm{c}} \mathrm{kg}^{-1}\right)$ & 0,05 \\
\hline $\mathrm{H}+\mathrm{Al}\left(\mathrm{cmol}_{\mathrm{c}} \mathrm{kg}^{-1}\right)$ & 1,32 \\
\hline $\mathrm{S}\left(\mathrm{cmol}_{\mathrm{c}} \mathrm{kg}^{-1}\right)$ & 2,51 \\
\hline $\mathrm{T}\left(\mathrm{cmol}_{\mathrm{c}} \mathrm{kg}^{-1}\right)$ & 3,83 \\
\hline $\mathrm{V}(\%)$ & 66 \\
\hline M.O. $\left(\mathrm{g} \mathrm{kg}^{-1}\right)$ & 6,0 \\
\hline Carbono $\left(\mathrm{g} \mathrm{kg}^{-1}\right)$ & 3,5 \\
\hline $\mathrm{C} / \mathrm{N}$ & 12 \\
\hline C.E. $\left(\mathrm{dS} \mathrm{m}^{-1}\right)$ & 0,30 \\
\hline Densidade do solo $\left(\mathrm{Mg} \mathrm{m}^{-3}\right)$ & 1,38 \\
\hline Densidade de Partícula ( $\left.\mathrm{Mg} \mathrm{m}^{-3}\right)$ & 2,59 \\
\hline Areia Grossa $\left(\mathrm{g} \mathrm{kg}^{-1}\right)$ & 370 \\
\hline Areia Fina $\left(\mathrm{g} \mathrm{kg}^{-1}\right)$ & 320 \\
\hline Silte $\left(\mathrm{g} \mathrm{kg}^{-1}\right)$ & 120 \\
\hline Argila $\left(\mathrm{g} \mathrm{kg}^{-1}\right)$ & 190 \\
\hline Classificação textural & Franco arenosa \\
\hline
\end{tabular}

(1) Análise física equímica do solo, segundo método da EMBRAPA (1997). 
impedir a deformação dos anéis durante o processo de compactação. A obtenção da coluna de solo compactada foi realizada em quatro etapas semelhantes, a partir de subamostras em volume necessário para a formação de um bl oco compactado com espessura final de $7 \mathrm{~cm}$.

A seguir, este bl oco teve a sua espessura reduzida em $1 \mathrm{~cm}$ em cada face, fazendo-se uso de um arame de aço, seguido da escarificação das col unas. O bloco compactado foi, então, retirado da camisa de ferroe do anel usado para compactação, tendo toda a sua circunferência aparada, de modo que o seu diâmetro fossereduzido em $0,5 \mathrm{~cm}$. A pós obter os quatro blocos de $5 \mathrm{~cm}$ de espessura necessários para formar a camada compactada de $20 \mathrm{~cm}$ de que compõe cada tratamento, os blocos foram col ocados dentro do anel de PVC, sendo a sua interface solo-PVC preenchida com pequena quantidade de caulim umedecido, compactado com bastão, até total preenchimento. Este procedimento teve por objetivo impedir que as raízes penetrassem nessa região, desviando da camada compactada e, assim, atingindo a camada inferior do conjunto, o quejá foi verificado em testes preliminares.

Para o preenchimento de todos os anéis, utilizaram-se amostras de solo que passou em peneira com malha de $4 \mathrm{~mm}$ de abertura e ficaram retidas na peneira de $2 \mathrm{~mm}$, previamenteumedeci das efertilizadas.

As colunas de solo foram expostas às condições naturais, sem qual quer tipo de proteção, ecultivadas com mudas de cajueiro anão precoce, clone CP-76, produzidas no Campo Experimental de Pacajus, pertencente ao Centro Nacional de Pesquisa da Agroindústria Tropical (CNPAT-E MBRAPA).

Durante o experimento, foram coletadas temperaturas do solo com o auxilio de três termômetros colocados aleatoriamente, em cada tratamento de isolamento térmico, a $15 \mathrm{~cm}$ de profundidade. As leituras de temperatura foram feitas diariamente às 8 e às $14 \mathrm{~h}$. A temperatura para o tratamento com isolamento térmico variou de $22^{\circ}$ a $36,6^{\circ} \mathrm{C}$ e, para o tratamento sem isol amento térmico, de 22,8 a 39,2 ${ }^{\circ} \mathrm{C}$.

Após o período de 12 meses de cultivo das plantas, a parte aérea foi colhida, determinando-se matéria seca da parte aérea (MSPA), altura da planta (AP), diâmetro do caule (DC) e número de ramificações da parte aérea (NRPA).

Os anéis da coluna de sol o foram separados e as raízes coletadas, determinando-se, por meio do número de pixels (NP), a área radicular (AR) e o comprimentoderaízes (CR). Para estas determinações, foram efetuados o processamento e a análise das imagens digitais com auxilio do Sistema I ntegrado para Análises de Raízes e Cobertura do Solo (SIARCS 3.0), desenvolvido peloCNPDIA-EMBRAPA, seguindo os procedimentos descritos por J orge \&
Crestana (1996) e J orge et al. (1996). Para obter as imagens, foi utilizado um scanner de mesa, com o uso de uma cuba de vidro de $5 \mathrm{~cm}$ de altura, $18 \mathrm{~cm}$ de largura e $36 \mathrm{~cm}$ de comprimento. Nesta cuba, as raízes lavadas foram imersas em água, evitando-se a sobreposição de imagens, e colocadas o mais perpendicular possível em relação à passagem da lâmpada do scanner, objetivando restringir a formação desombras. A entrada deluz pelas laterais foi evitada com a construção de uma moldura de isopor. Foi determinada, ainda, a densidaderadicular, calculada por meio da relação comprimento radicular/volume desolo no qual seencontravamessas raízes, bem como a matéria seca de raízes.

Realizou-se a análise de variância (ANOVA) dos dados coletados, pelo teste $\mathrm{F}$ a $5 \%$. A comparação de médias foi feita pelo teste Student-NewmanKeuls (SNK), a $5 \%$. Equações de regressão foram ajustadas aos dados.

\section{RESULTADOS E DISCUSSÃO}

A temperatura do solo afetou a altura das plantas (AP), produção de matéria seca da parte aérea (MSPA) e o diâmetro do caule (DC) (Quadro 2). Entretanto, não teve efeito sobre o número de ramificações (NRPA), enquanto a densidade do solo afetou somente a altura das plantas (AP). A interação das variáveis foi significativa somente para o número de ramificações (NRPA).

Os resultados apresentados no quadro 3 mostram que, independentemente da densidade do solo, as médias foram superiores no TI em relação ao TNI, para as variáveis AP, MSPA e DC. De acordo com Barros et al . (1993), o cajuei ro suporta temperaturas médias de até $35^{\circ} \mathrm{C}$, sendo a média de $27^{\circ} \mathrm{C}$ a mais apropriada. Considerando as temperaturas máximas de 36,6 e $39,2^{\circ} \mathrm{C}$, para oTI eTNI, obtidas com a leitura diária, bem como o fato de que, noTNI, o número de horas nestes níveis máximos possa ter ocorrido por mais tempo, é provável que as temperaturas devam ter atingido níveis limitantes, principalmente no TNI, para alguns processos fisiológicos da planta, comprometendo o crescimento radicular e a absorção de íons e água, como observado. Sidiras \& Pavan (1985), Bragagnolo \& Miel niczuk (1990) e Salton \& Mielniczuk (1995), trabalhando com diferentes sistemas de manejo do solo e culturas, também fizeram constatações semelhantes.

Ao avaliar as alturas das plantas de cajueiro, verificou-se que elas apresentaram uma redução linear com o aumento dos níveis de compactação do solo (Figura 1), fato também observado em soja (Borges et al., 1988), em capim-gordura e colonião (Silva et al., 1992), em feijão-de-porco e em feijãobravo-do-Ceará (Alvarenga, 1993), cultivados em colunas de PVC. 
Quadro 2. Quadrados médios das variáveis altura das plantas (AP), diâmetro do caule (DC), matéria seca da parte aérea (MSPA), e número de ramificações (NRPA) do cajueiro cultivado em colunas de solo com diferentes níveis de densidade e temperatura do solo

\begin{tabular}{lccccc}
\hline Causa de variação & GL & AP & DC & MSPA & NRPA \\
\hline Temperatura do solo (T) & 1 & $393,36^{* *}$ & $4,69^{*}$ & $1456,69^{* *}$ & $0,25^{\text {ns }}$ \\
Densidade do solo (D) & 5 & $61,42^{* *}$ & $0,29^{\text {ns }}$ & $43,917^{\text {ns }}$ & $0,25^{\text {ns }}$ \\
T x D & 5 & $24,36^{\text {ns }}$ & $0,29^{\text {ns }}$ & $30,228^{\text {ns }}$ & $1,98^{* *}$ \\
Resíduo & 24 & 14,02 & 0,11 & 30,472 & 0,38 \\
C.V. (\%) & & 13,39 & 24,49 & 39,20 & 35,63
\end{tabular}

*, ** Significativos a 5 e $1 \%$, respectivamente, pelo teste $\mathrm{F}$.

As ramificações da parte aérea das plantas de cajueiro cultivadas com isolamento térmico apresentaram comportamento cúbico de acordo com o aumento da densidade do solo, enquanto essa variável sem isolamento térmico nãofoi influenciada pela densidade do solo (Figura 2). Estes resultados demonstram ter havido um aumento do número de ramificações a partir da densidade de $1,4 \mathrm{Mg} \mathrm{m}^{-3}$,

Quadro 3. Altura de plantas (AP), diâmetro do caule (DC) e produção de matéria seca da parte aérea (MSPA) do cajueiro cultivado em colunas de solo com diferentes níveis de temperatura

\begin{tabular}{crrr}
\hline Tratatamento térmico & AP & DC & MSPA \\
\hline TI & $31 \mathrm{a}$ & $1,83 \mathrm{a}$ & $20 \mathrm{a}$ \\
TNI & $25 \mathrm{~b}$ & $1,00 \mathrm{~b}$ & $8 \mathrm{~b}$
\end{tabular}

Médias seguidas pela mesma letra, na coluna, não diferem entre si, pel o teste F, a $5 \%$; TI: tratamento isolado; TNI: tratamento não isolado. seguido de um decréscimo a partir da densidade $1,7 \mathrm{Mg} \mathrm{m}^{-3}$. É possível que este comportamento esteja associado com a maior restrição mecânica ao crescimento radicular, na camada compactada, como confirma Alvarenga (1993), favorecendo o crescimentolateral das plantas. Contudo, a redução do número de ramificações a partir da densidade de $1,7 \mathrm{Mg} \mathrm{m}^{-3}$ indica que, na presença de altos valores de densidade, as plantas reduziram também o seu crescimentolateral.

Quando se avaliou o NRPA em cada tratamento de densidade, contudo, verificou-se que esta variável foi semelhante à da maioria dos tratamentos testados, excetuando-se para a densi dade $1,6 \mathrm{Mg} \mathrm{m}^{-3}$, indicando maior número de ramificações no tratamento que proporcionou temperaturas do solo mais baixas (TI) (Quadro 4).

\section{Crescimento do sistema radicular}

Verificou-se que a temperatura influenciou as variáveis estudadas (Quadro 5), reduzindo sensivelmente o desenvolvimento do sistema radicular das plantas que não foram submetidas ao isolamento térmico. Rosolem et al. (1992) observaram que o

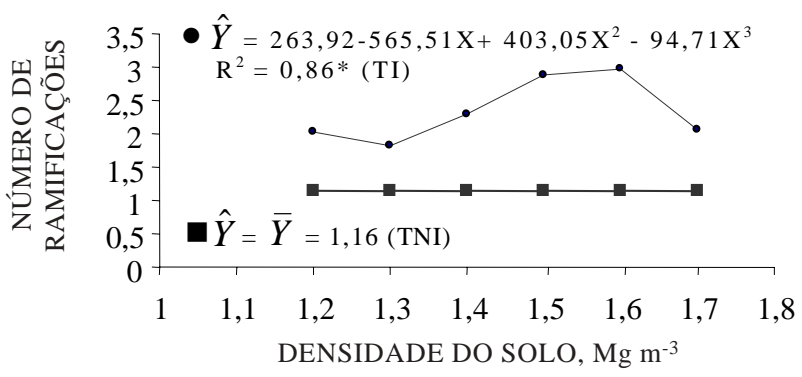

Figura 2. Número de ramificações da parte aérea de plantas de cajueiro anão precoce, cultivadas em colunas de solo com tratamentos térmicos; isolado (TI) e não isolado (TNI), considerando a densidade do solo. 
crescimento do sistema radicular é resultante da interação de fatores quími cos e físi cos do solo, clima e características da planta. Sidiras \& Pavan (1986), comparando resultados obtidos em diferentes sistemas de manejo do solo, verificaram que, nos sistemas de manejo sem proteção do solo, ocorreram temperaturas inadequadas para a cultura da soja.

O impedimento subsuperficial, caracterizadopela camada compactada no segundo anel, levou o cajueiro a acumular mais raízes na camada superficial da coluna, onde as condições físicas do solo apresentavam-se mais adequadas, resultado semel hante ao encontrado por Cintra \& Mielniczuk (1983) e Borges et al. (1988), em soja, e Silva et al. (1992), em gramíneas forrageiras. O ajustede equações indicou este comportamento para as variáveis em estudo área radicular e comprimento de raízes (Quadro 6 e Figura 3).

Os resultados alcançados no segundo anel (camada compactada), onde as variáveis área radicular, comprimento radicular e matéria seca do sistema radicular não se diferiram, excetuando-sea densidade radicular, com comportamento cúbico (Quadro 6 e Figura 3), parecem indicar que a produção de raízes, nesta camada, ocorreu de forma irregular entre os diferentes níveis de compactação. Este comportamento está associado às características físicas de redução da macroporosidade e aumento da resistência à penetração do solo, como é reconhecido também por Borges et al. (1988). No terceiro anel, somente a matéria seca do sistema radicular apresentou ajuste, sendo de comportamento quadrático. Este fato foi também constatado no anel 4 para esta variável, bem como para comprimento de raízes, enquanto que as demais não apresentaram qualquer tipo de ajuste, tanto no anel 3 quanto no 4.

O comportamento das raízes nos anéis inferiores (3 e 4) mostrou que o maior acúmulo de raízes, ocorrido à medida que aumentava a densidade do solo, influenciou o decréscimo de raízes dos anéis inferiores, como mostram, de forma mais clara, as equações correspondentes a comprimento radicular edensidade radicular no anel 4. Observou-se, ainda,

Quadro 4. Número de ramificações da parte aérea de plantas de cajueiro anão precoce, cultivadas em colunas de solo com tratamentos térmi cos; isolado (TI) e não isolado (TNI ), considerando a densidade do solo

\begin{tabular}{|c|c|c|c|c|c|c|}
\hline \multirow{2}{*}{ Tratamento térmico } & \multicolumn{6}{|c|}{ Densidade } \\
\hline & 1,2 & 1,3 & 1,4 & 1,5 & 1,6 & 1,7 \\
\hline & \multicolumn{6}{|c|}{$-\mathrm{Mg} \mathrm{m}^{-3}$} \\
\hline $\mathrm{TI}$ & $2,00 \mathrm{a}$ & $2,00 \mathrm{a}$ & $2,00 \mathrm{a}$ & $3,00 \mathrm{a}$ & $3,00 \mathrm{a}$ & $2,00 \mathrm{a}$ \\
\hline TNI & $1,00 \mathrm{a}$ & $1,00 \mathrm{a}$ & $2,00 a$ & $1,00 \mathrm{a}$ & $1,00 \mathrm{~b}$ & $1,00 \mathrm{a}$ \\
\hline
\end{tabular}

Médias seguidas pela mesma letra, na coluna, não diferem entre si, pelo teste de SNK, a 5 \%.

Quadro 5. Área radicular (AR), comprimento de raízes (CR), matéria seca de raízes (MSR ) e densidade radicular (DR) de plantas de cajueiro cultivadas em colunas de solo com diferentes níveis de compactação e submetidas a tratamentos térmicos; isolado (TI) e não isolado (TNI)

\begin{tabular}{cccccc}
\hline Tratamento térmico & Anel & AR & CR & MSR & DR \\
\hline & & $\mathrm{cm}^{2}$ & $\mathrm{~m}$ & $\mathrm{~g}$ & $\mathrm{~cm} \mathrm{~cm}^{-3}$ \\
TI & 1 & $93,47 \mathrm{a}$ & $43,33 \mathrm{a}$ & $9,67 \mathrm{a}$ & $1,23 \mathrm{a}$ \\
TNI & $25,47 \mathrm{~b}$ & $10,96 \mathrm{~b}$ & $2,61 \mathrm{~b}$ & $0,31 \mathrm{~b}$ \\
TI & 2 & $59,09 \mathrm{a}$ & $27,55 \mathrm{a}$ & $5,04 \mathrm{a}$ & $0,78 \mathrm{a}$ \\
TNI & $24,90 \mathrm{~b}$ & $11,21 \mathrm{~b}$ & $1,61 \mathrm{~b}$ & $0,32 \mathrm{~b}$ \\
TI & 3 & $50,56 \mathrm{a}$ & $26,85 \mathrm{a}$ & $2,50 \mathrm{a}$ & $0,76 \mathrm{a}$ \\
TNI & & $18,41 \mathrm{~b}$ & $8,23 \mathrm{~b}$ & $0,94 \mathrm{~b}$ & $0,23 \mathrm{~b}$ \\
TI & 4 & $68,00 \mathrm{a}$ & $34,35 \mathrm{a}$ & $3,14 \mathrm{a}$ & $0,97 \mathrm{a}$ \\
TNI & & $22,19 \mathrm{~b}$ & $10,34 \mathrm{~b}$ & $0,84 \mathrm{~b}$ & $0,29 \mathrm{~b}$ \\
\end{tabular}

Médias seguidas pela mesma letra, na coluna, não diferem estatisticamente entre si, pelo teste F, a $5 \%$. 
ter havido uma tendência de algumas plantas compensarem a limitação de crescimento radicular na camada compactada, aumentando a quantidade de raízes abaixo dessa camada, o que verifica-se na produção de matéria seca do sistema radicular (MSR) no anel 4 (Quadro 6 e Figura 3). Esse resultado também foi observado por Alvarenga (1996) para algumas leguminosas.

Quadro 6. Equações de regressão ajustadas para área radicular (AR), comprimento de raízes (CR ), matéria seca de raízes (MSR), densidade radicular (DR ), considerando os níveis de compactação do solo nas quatro camada do solo estudadas

\begin{tabular}{|c|c|c|c|}
\hline Anel & Variável & E quação & $\mathbf{R}^{2}$ \\
\hline \multirow[t]{4}{*}{1} & $A R$ & $\hat{Y}=-120,00+131,40 X$ & $0,80 *$ \\
\hline & $\mathrm{CR}$ & $\hat{Y}=383,91-574,57 \mathrm{X}+227,18 \mathrm{X}^{2}$ & $0,92 *$ \\
\hline & MSR & $\hat{Y}=\bar{Y}=6,69$ & - \\
\hline & $\mathrm{DR}$ & $\hat{Y}=\bar{Y}=0,08$ & - \\
\hline \multirow[t]{4}{*}{2} & $A R$ & $\hat{Y}=\bar{Y}=34,17$ & - \\
\hline & $\mathrm{CR}$ & $\hat{Y}=\bar{Y}=17,00$ & - \\
\hline & MSR & $\hat{Y}=\bar{Y}=1,45$ & - \\
\hline & $\mathrm{DR}$ & $\hat{Y}=-0,10+0,22 \mathrm{X}-0,15 \mathrm{X}^{2}+0,35 \mathrm{X}^{3}$ & $0,82^{*}$ \\
\hline \multirow[t]{4}{*}{3} & $A R$ & $\hat{Y}=\bar{Y}=36,93$ & - \\
\hline & $\mathrm{CR}$ & $\hat{Y}=\bar{Y}=15,90$ & - \\
\hline & MSR & $\hat{Y}=73,26-97,20 \mathrm{X}+32,56 \mathrm{X}^{2}$ & $0,80 *$ \\
\hline & $\mathrm{DR}$ & $\hat{Y}=\bar{Y}=0,05$ & - \\
\hline \multirow[t]{4}{*}{4} & $A R$ & $\hat{Y}=\bar{Y}=41,70$ & - \\
\hline & $\mathrm{CR}$ & $\hat{Y}=265,46-319,03 \mathrm{X}+101,98 \mathrm{X}^{2}$ & $0,76^{*}$ \\
\hline & MSR & $\hat{Y}=95,22-134,42 X+47,92 X^{2}$ & $0,90 *$ \\
\hline & $\mathrm{DR}$ & $\hat{Y}=\bar{Y}=0,06$ & - \\
\hline
\end{tabular}

Significativo a $5 \%$, pelo teste $\mathrm{F}$.
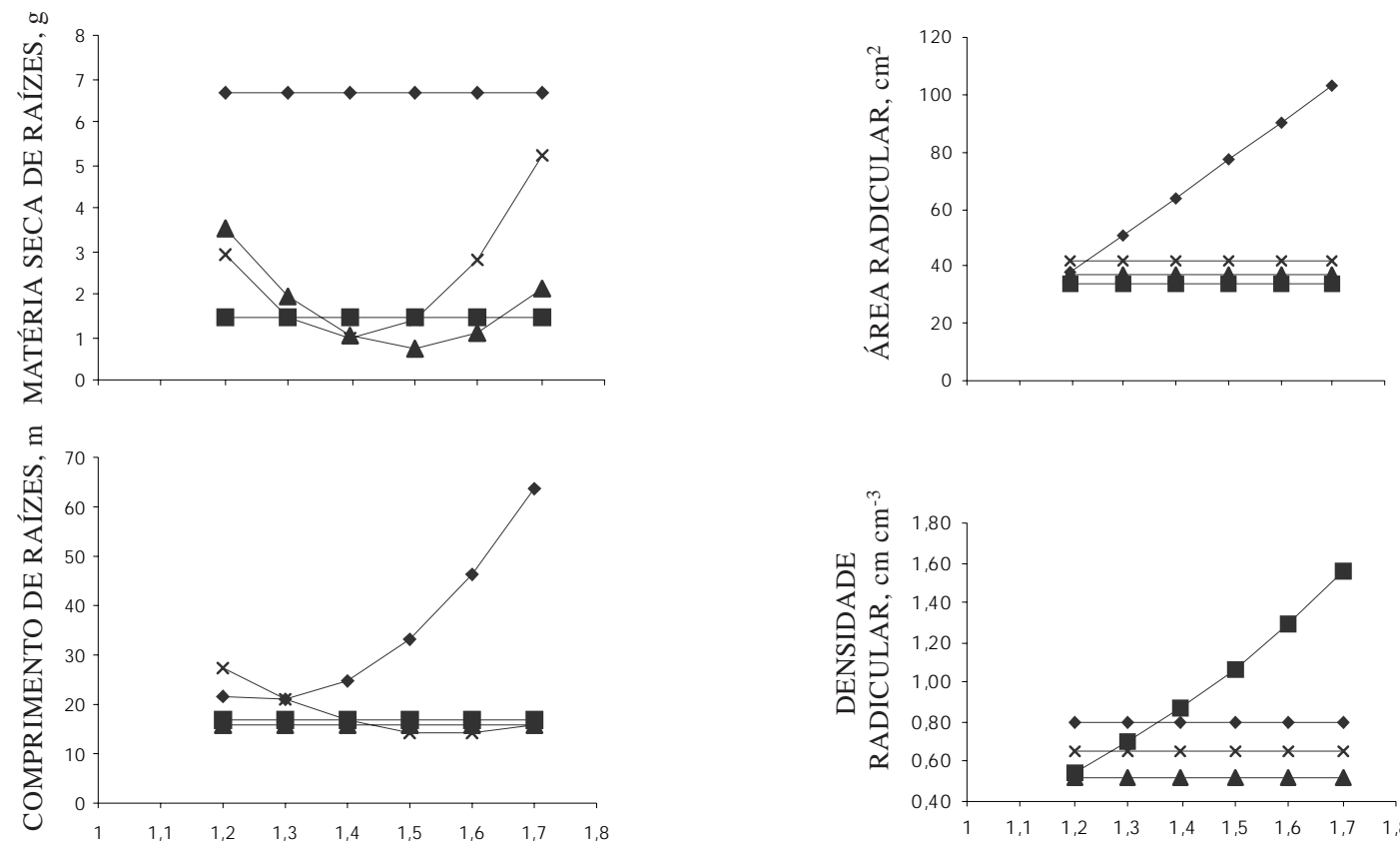

DENSIDADE DO SOLO, $\mathrm{Mg} \mathrm{m}^{-3}$

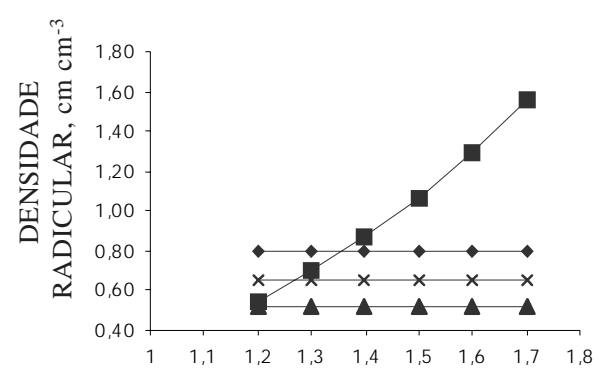

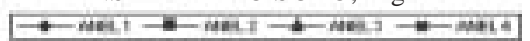

Figura 3. Matéria seca de raízes (MSR), área radicular (AR), comprimento de raízes (CR), densidade radicular (DR) de plantas de cajueiro cultivadas em colunas de solo, considerando diferentes níveis de densidade do solo. 


\section{CONCLUSÕES}

1. O aumento de temperatura reduziu o crescimento vegetativo, afetando tanto a parteaérea quanto o sistema radicular.

2. O aumento dos níveis de compactação do solo reduziu o crescimento da parte aérea das plantas de cajueiro e impediu a penetração das raízes abaixo da camada compactada.

\section{AGRADE CIMENTOS}

Os autores agradecem ao Centro Nacional de Pesquisa da Agroindústria Tropical (CNPAT) da EMBRAPA, pela colaboração na realização deste trabalho.

\section{LITE RATURA CITADA}

ALVARENGA, R.C. Potencialidades de adubos verdes para conservação e recuperação de solos. Viçosa, U niversidade Federal de Viçosa, 1993. 112p. (Tese de Doutorado)

ALVARENGA, R.C.; COSTA, L.M.; MOURA FILHO, W. \& REGAZZI, A.J . Crescimento de raízes de leguminosas em camadas de solo compactadas artificialmente. R. Bras. Ci. Solo, 20:319-326, 1996.

ARAÚ J O, J.P.P. Situação atual e perspectivas da cajucultura brasileira. Fortaleza, EMBRAPA-CNPCa, 1991. não paginado

BARROS, L.M.; PIMENTEL, C.R.M.; CORREA, M.P.F. \& MESQUITA, A.L.M. Recomendações técnicas para a cultura do cajueiro-anão-precoce. Fortaleza, EMPRAPACNPAT, 1993. 65p. (Circular Técnica, 1)

BORGES, E.N.; NOVAIS, R.F.; REGAZZI , A.J .; FERNANDES, B. \& BARROS, N.F. Respostas de variedades de soja à compactação de camadas de sol o. R. Ceres, 35:553-568, 1988.

BORTOLUZZI, E.C. \& ELTZ, F.L.F. Efeito do manejo mecânico da palhada de aveia preta sobre a cobertura, temperatura, teor deágua no solo eemergência da soja em sistema plantio direto. R. Bras. Ci. Solo, 24:449-457, 2000.

BRADY, N.C. Natureza e propriedades do solo. 2.ed. Rio de J aneiro, Freitas Bastos, 1989. 878p.

BRAGAGNOLO, N. \& MIELNICZUK, J. Cobertura do solo por palha de trigo e seu relacionamento com a temperatura e umidade do solo. R. Bras. Ci. Solo, 14:369-374, 1990.
CINTRA, F.L.D. \& MIELNICZUK, J. Potencial de algumas espécies vegetais para recuperação de solos com propriedades físicas degradadas. R. Bras. Ci. Solo, 7:197201, 1983.

EMPRESA BRASILEIRA DE PESQUISA AGROPECUÁRIA EMBRAPA. Centro Nacional de Pesquisa deSolos. Manual de métodos de análise de solo. 2.ed. Rio de J aneiro, 1997. $212 p$.

J ORGE, L.A.C. \& CRESTANA, S. Recomendações práticas para utilização do SIARCS 3.0 nos estudos de raízes, cobertura vegetal, fol has e outras aplicações. São Carlos, EMBRAPACNPDIA, 1996. (Recomendação Técnica, 4)

J ORGE, L.A.C.; RALISCH, R.; SAAB, O.J.G.; MEDINA, C.C.; GUIMARÃES, M.F.; NEVES, C.S.V.J .; CRESTANA, S. CINTRA, F.L.D.; BASSOI, L.H. \& FERNANDES, S.B.V. Aquisição de imagens de raízes. In: J ORGE, L.A.C. Recomendações práticas para aquisição de imagens digitais analisadas através do SIARCS ${ }^{\circledR}$. São Carlos, EMBRAPACNPDIA, 1996. (Circular Técnica, 1)

MERTEN, G.H. \& MIELNICZUK, J. Distribuição do sistema radicular e dos nutrientes em Latossolo Roxo sob dois sistemas de preparo de solo. R. Bras. Ci. Solo, 15:369374, 1991.

MOTA, F.S. Meteorologia agrícola. 3.ed. São Paulo, Nobel, 1977. $376 p$.

PAULA PESSOA, P.F.A.; LEITE, L.A.S. \& PIMENTEL, C.R.M. Situação atual e perspectivas da agroindústria do caju. In: ARAÚJ O, J .P.P. \& SILVA, V.V., eds. Cajucultura: modernas técnicas de produção. Fortaleza, EMBRAPA-CNPAT, 1995. p.23-42.

PIMENTEL, C.R.M.; PAULA PESSOA, P.F.A. \& LIMA, L.A.A. Análise estrutural e disponibilidade de tecnologia para a cajucultura brasileira. Fortaleza, EMBRAPA-CNPAT, 1993. 31p. (Documentos, 8)

ROSOLEM, C.A.; FURLANI J r., E.; BICUDO, S.J .; MOURA, E.G. $\&$ BULHÕES, L.H. Preparo do solo e sistema radicular do trigo. R. Bras. Ci. Solo, 16:115-120, 1992.

SALTON, J.C. \& MIELNICZUK, J. Relações entre sistemas de preparo, temperatura eumidade de um Podzólico Vermel hoEscuro de Eldorado do Sul (RS). R. Bras. Ci. Solo, 19:313319, 1995.

SIDIRAS, N. \& PAVAN, M.A. Influência do sistema de manejo do solo no seu nível de fertilidade. R. Bras. Ci. Solo, 9:249-254, 1985.

SILVA, G.P.; NOVAIS, R.F.; NEVES, J.C.L. \& BARROS, N.F. Respostas de espécies de gramíneas a camadas compactadas de solo. R. Ceres, 39:31-43, 1992. 